\title{
Identification and evaluation of PCR reference genes for host and pathogen in sugarcane-Sporisorium scitamineum interaction system
}

Ning Huang ${ }^{1}$, Hui Ling ${ }^{1}$, Feng Liư ${ }^{1}$, Yachun Su, ${ }^{1}$, Weihua Su${ }^{1}$, Huaying Mao ${ }^{1}$, Xu Zhang ${ }^{1}$, Ling Wang ${ }^{1}$, Rukai Chen ${ }^{1}$ and Youxiong Que ${ }^{1,2,3^{*}}$

\begin{abstract}
Background: Sugarcane (Saccharum L. plant) is an important crop for sugar and bio-energy production around the world. Among sugarcane diseases, smut caused by Sporisorium scitamineum is one of the major fungal diseases causing severe losses to the sugarcane industry. The use of PCR reference genes is essential to the normalization of data on gene expression involving the sugarcane-S. scitamineum interaction system; however, no report that addresses criteria in selecting these reference genes has been published to date.

Results: In this study, 10 sugarcane genes and eight S. scitamineum genes were selected as candidate PCR reference genes in the sugarcane-S. scitamineum interaction system. The stability and reliability of these 18 candidate genes were analyzed in smut-resistant (NC0376) and -susceptible (YC71-374) genotypes using the statistical algorithms geNorm, NormFinder, BestKeeper, and deltaCt method. Subsequently, the relative expression levels of the sugarcane chitinase I-3 gene and S. scitamineum chorismate mutase gene were determined to validate the applicability of these sugarcane and S. scitamineum PCR reference genes, respectively. We finally found that the acyl-CoA dehydrogenase gene (ACAD), serine/arginine repetitive matrix protein 1 gene (SARMp 1), or their combination (ACAD + SARMp1) could be utilized as the most suitable reference genes for normalization of sugarcane gene expression in sugarcane bud tissues after S. scitamineum infection. Similarly, the inosine 5'monophosphate dehydrogenase gene (S10), the SEC65-signal recognition particle subunit gene (S11), or their combination (S10 + S11) were suitable for normalization of S. scitamineum gene expression in sugarcane bud tissues.
\end{abstract}

Conclusions: The PCR reference genes ACAD, SARMp1, S10, and S11 may be employed in gene transcriptional studies involving the sugarcane-S. scitamineum interaction system.

Keywords: Saccharum L., Smut fungus, Quantitative real-time PCR, Reference gene

\footnotetext{
* Correspondence: queyouxiong@126.com

'Key Laboratory of Sugarcane Biology and Genetic Breeding, Ministry of Agriculture, Fujian Agriculture and Forestry University, Fuzhou 350002, China ${ }^{2}$ Key Laboratory of Ministry of Education for Genetics, Breeding and Multiple Utilization of Crops, College of Crop Science, Fujian Agriculture and Forestry University, Fuzhou 350002, China

Full list of author information is available at the end of the article
}

(c) The Author(s). 2018 Open Access This article is distributed under the terms of the Creative Commons Attribution 4.0 International License (http://creativecommons.org/licenses/by/4.0/), which permits unrestricted use, distribution, and reproduction in any medium, provided you give appropriate credit to the original author(s) and the source, provide a link to the Creative Commons license, and indicate if changes were made. The Creative Commons Public Domain Dedication waiver (http://creativecommons.org/publicdomain/zero/1.0/) applies to the data made available in this article, unless otherwise stated. 


\section{Background}

Regarding the development of technologies in biological research, omics data have led to a considerable increase in gene identification [1]. Gene expression analysis is essential in understanding the signaling and metabolic pathways that underly cellular and developmental processes [2]. Currently, real-time quantitative PCR (qRT-PCR), semi-quantitative PCR (semi-qPCR), and northern blotting are the major methods of quantifying and validating the expression of genes [3-6]. Reference genes, which are expressed stably in different organ tissues, at different developmental stages, or under specific-experimental conditions, can improve the precision of gene expression quantification by reducing experimental errors caused by RNA quality, cDNA synthesis, qPCR reactions, or other factors [7]. Previously, housekeeping genes, which were indispensable in maintaining basic metabolic activities and basic structural components, were widely used as PCR reference genes for gene expression analysis in humans [8], animals [9] and plants [10]. Furthermore, expression analysis of pathogen genes during infection and colonization in the host plant has been the main step in elucidating the biological function of pathogen genes. To reduce errors in expression quantification of pathogen genes caused by the adaptability of the pathogen, as well as nutrition and stress in host plants $[11,12]$, pathogen PCR reference genes have been included in investigations. However, there is growing evidence showing there is no single, universal gene that could be utilized in various experimental conditions [13], and the stability of reference genes should be validated before these are used for normalization of gene expression $[14,15]$.

Over the past decades, the evaluation of PCR reference genes has been reported in various plant species such as Oryza sativa [16], Zea mays [17], Brassica juncea [18], Triticum aestivum [19], and Nicotiana tabacum [20]. Sugarcane (Saccharum L.), which belongs to Gramineae, is an important crop for sugar and bio-energy production in more than 110 tropical and subtropical countries around the world [21]. Sugarcane PCR reference genes, which are used in the normalization of gene expression in different tissues of sugarcane varieties (glyceraldehyde-3-phosphate dehydrogenase, GAPDH) [22] or under different abiotic stresses (eukaryotic elongation factor 1A, eEF1A; cullin, CUL; and clathrin adaptor complex, $C A C)$ [23-25], have been evaluated and reported. PCR reference genes that are identified by commonly used algorithms geNorm [26], NormFinder [27], BestKeeper [28], and the deltaCt method [8] allow reliable and accurate normalization for gene expression data [29-32]. Analysis using geNorm is based on two reference genes that would show highly identical expression pattern across different experimental biological samples [26]. NormFinder is a mathematical algorithm that estimates gene expression stability by comparing variations of gene expression in intra/inter-groups within a group or groups of bio-samples [27]. BestKeeper assesses the expression stability of genes by employing Pearson, Spearman, and Kendall Tau correlation coefficients for pair-wise correlation analysis, generating weighted indices of the candidate genes [28]. The deltaCt method [26] describes the $\Delta \mathrm{Ct}$ approach, which requires less use of specialist programs and biomaterials by comparing pairs of candidate genes [8]. With increasing demand for sugarcane production, a growing number of RNA-seq trials have been conducted to identify genes that are associated with specific biological processes such as sugar accumulation [33], fiber content [34], and stress responses [3, 4, 23-25]. Smut disease, which is caused by Sporisorium scitamineum, is one of the major fungal affecting sugarcane growth, often resulting in a $3 \%-7 \%$ reduction in sugar content [21]. Wu et al. [4] identified 2015 sugarcane differentially expressed ESTs using the Solexa sequencing technology, and Que. et al. [3] identified a total of 65,852 sugarcane unigenes by RNA sequencing during $S$. scitamineum infection. Genome sequencing has indicated that the genome of $S$. scitamineum, which is $19.63 \mathrm{Mb}$ and $19.98 \mathrm{Mb}$ in size, consists of about 6636 to 6693 genes, which include 527 secreted protein genes, 192 pathogenic genes, and 68 effector protein genes [35-37]. Yan et al. [38] identified 52 genes that were regulated by the b-locus by de novo RNA-sequencing of $S$. scitamineum and its resultant $S s \triangle M A T-1 b$ mutant. By comparing the transcriptome of S. scitamineum in sugarcane tissues and in vitro, Taniguti et al. [36] identified 125 differentially expressed genes at $5 \mathrm{~d}$ and 907 differentially expressed genes at $200 \mathrm{~d}$. Although numerous sequences of differentially expressed genes have been isolated from sugarcane and S. scitamineum after infection [4, 36, 38-41], details of the underlying regulatory network remain unclear. Expression profiling of defense-related genes in sugarcane and pathogenesis-related genes in S. scitamineum is essential to the elucidation of the molecular basis of the sugarcane-S. scitamineum interaction system. To date, no report on reference gene selection for gene expression normalization in sugarcane-S. scitamineum interaction system has been published.

In this study, 10 sugarcane candidate PCR reference genes were obtained, including six (acyl-CoA dehydrogenase, $A C A D$; casein kinase I isoform delta-like, $C K 1 \delta$; OTU domain-containing protein 5 , OTU5; 12-oxophytodienoate reductase 7, OPR7; polyadenylate-binding protein $8, P A B P 8$; serine/arginine repetitive matrix protein $1, S A R M p 1$ ) from our previous transcriptomic data [3] and four (GAPDH, $e E F 1 A, C U L$, and $C A C$ ) from previous reports [23, 24]. In addition, eight $S$. scitamineum candidate PCR reference genes (conserved hypothetical protein, S2; conserved hypothetical protein, S4; VPS73-protein involved in vacuolar 
protein sorting, S6; synaptobrevin, S8; GTP-binding protein Rac1, S9; inosine 5'-monophosphate dehydrogenase, S10; SEC65-signal recognition particle subunit, S11; and ADP-ribosylation factor, S12) were selected from a $S$. scitamineum genomic-wide expression profile microarray (unpublished, Huang et al., hning2012@126. com). The expression of the 18 candidate PCR reference genes in smut-infected buds of smut-susceptible genotype YC71-374 and smut-resistant genotype NCo376 was assessed using qRT-PCR, and their stabilities were evaluated using algorithms geNorm, NormFinder, BestKeeper, and the deltaCt method. This study aimed to identify suitable PCR reference genes for accurate normalization and quantification of gene expression levels of the host and pathogen in a sugarcane-S. scitamineum interaction system.

\section{Methods}

\section{Plant materials and treatment}

The smut-resistant genotype NCo376 and the -susceptible genotype YC71-374 were provided by the Key Laboratory of Sugarcane Biology and Genetic Breeding, Ministry of Agriculture, Fujian Agriculture and Forestry University. The disease-free sugarcane materials were collected and cut into single bud canes, immersed in $5 \mathrm{~g} / \mathrm{L}$ carbendazim (Shanghai Huanong Chemical Co. Ltd., Shanghai, China) for $48 \mathrm{~h}$, and then transferred into a $50{ }^{\circ} \mathrm{C}$ water bath for $2 \mathrm{~h}$. The buds were embedded in sterile nutritional soil in an incubator with $28+0.5^{\circ} \mathrm{C}$, $3000 \mathrm{~lx}$, and $16 \mathrm{~h}$ light $/ 8 \mathrm{~h}$ dark conditions. After incubation for $7 \mathrm{~d}$ to $10 \mathrm{~d}$, the buds grew to a length of 1 $2 \mathrm{~cm}$ and were divided into two groups, namely, the control group and experimental group. And then these two groups were injected with $0.5 \mu \mathrm{L}$ sterile water $(0.01 \% \mathrm{~V} / \mathrm{V}$, Tween-20/water) and $0.5 \mu \mathrm{L}$ of a $S$. scitamineum spores suspension (density: $5 \times 10^{6} / \mathrm{mL}, 0.01 \% \mathrm{v} / \mathrm{v}$, Tween 20/water) respectively, and placed into the same incubator. Both groups consisted of three biological replicates. Each sample included five sugarcane buds, which were excised for RNA extraction at $0 \mathrm{~d}, 3 \mathrm{~d}$, and $7 \mathrm{~d}$ after injection. All samples were immediately frozen in liquid nitrogen and stored at $-80{ }^{\circ} \mathrm{C}$ until use.

\section{RNA isolation and CDNA synthesis}

RNA was isolated using TRIzol (Invitrogen, Shanghai, China) and assessed in quantity and quality using a multifunction microplate reader Synergy H1 (Bio-Tek, Winooski, VT, USA) and 1.5\% agarose gel electrophoresis. Prior to cDNA synthesis, any contaminating genomic DNA in the total RNA samples was removed using RNase-free DNase I (Promega, WI, USA). cDNA was synthesized using a PrimeScript RT kit (Perfect for Real Time) (TaKaRa Biotech., Dalian, China) following the manufacturer's recommendations, resolved using 1.5\% agarose gel electrophoresis, and then stored at $-20{ }^{\circ} \mathrm{C}$. To exclude the genomic DNA contaminating cDNA samples, all the cDNA samples were detected by the SD7R primers (ACTTACGAGCACCTCAGGGA/AGAGTCCGAAGCC GAAGAT) before use it as qRT-PCR template, which could achieve two fragments in genomic DNA samples and one fragment in cDNA samples [42].

\section{Identification of candidate PCR reference genes and primer design}

$G A P D H, e E F 1 A, C U L$, and $C A C$ were chosen as sugarcane candidate PCR reference genes in the present study based on previous reports [23, 24]. The other six sugarcane candidate PCR reference genes $(A C A D$, CK1 $\delta$, OTU5, OPR7, PABP8, and SARMp1), which were expressed stably at higher levels than that of GAPDH in the transcriptomic data of sugarcane under S. scitamineum stress [3], were also chosen. Similarly, based on the expression profile of $S$. scitamineum genes in the genome-wide expression profile microarray (unpublished, Huang et al., hning2012@126.com), eight S. scitamineum genes (S2, S4, S6, S8, S9, S10, S11, and S12) with high and stable expression levels were selected as S. scitamineum candidate PCR reference genes. Furthermore, except for $e E F 1 A$, CUL, and CAC [23], the qRT-PCR primers of all the other candidate PCR reference genes were designed using Primer-BLAST (https://www.ncbi.nlm. nih.gov/tools/primer-blast/) and presented in Table 1.

\section{qRT-PCR and data analysis}

qRT-PCR was prepared using the SYBRGreen Universal Master Mix kit (Roche, NY, USA) and consisted of a cDNA template (equivalent to 10 ng of RNA), primers, and $\mathrm{ddH}_{2} \mathrm{O}$. Each qPCR reaction contained three technical replicates and used $\mathrm{dd}_{2} \mathrm{O}$ as a blank control. The qRT-PCR amplification program was as follows: $50^{\circ} \mathrm{C}$ for $2 \mathrm{~min}, 95^{\circ} \mathrm{C}$ for $10 \mathrm{~min}$, followed by 40 cycles of $95^{\circ} \mathrm{C}$ for $15 \mathrm{~s}, 60{ }^{\circ} \mathrm{C}$ for $1 \mathrm{~min}$, which were the default parameters of the ABI 7500 FAST Real-Time PCR System. Using a series of gradient-diluted cDNA samples as the template, the $\mathrm{Ct}$ value of each primer pair was calculated after qRT-PCR analysis and was used to generate a standard curve, following the amplification efficiency of each primer pair [23].

In the present study, $36 \mathrm{cDNA}$ samples $(0 \mathrm{~d}, 3 \mathrm{~d}$, and 7 $\mathrm{d}$ samples in the experimental group and the control group, including three biological replicates) were used to assess the expression of 10 sugarcane candidate PCR reference genes. For undetection or inexistence of S. scitamineum cells in the $0 \mathrm{~d}$ samples in the experimental and the control groups, only $3 \mathrm{~d}$ and $7 \mathrm{~d}$ samples in the experimental group (12 cDNA samples, each sample includes three biological replicates) were used for assessing the eight $S$. scitamineum candidate PCR reference genes. The 
Table 1 Primers of candidate PCR reference genes

\begin{tabular}{|c|c|c|}
\hline Category & Gene Name & Sense Primer/Anti-sense Primer ( $\left.5^{\prime}-3^{\prime}\right)$ \\
\hline Sugarcane & Acyl-CoA dehydrogenase family member $10, A C A D$ & CGTGGCATGGATCTGATGGT/AGCCTGCTCCAGTTCAATCC \\
\hline Sugarcane & Casein kinase I isoform delta-like, $C K 1 \delta$ & TCAAGGGCTACCTCCCTCTC/GCATTCTTCCCTTCCGCTCT \\
\hline Sugarcane & OTU domain-containing protein 5, OTU5 & TGGTGCAGAGCCCATTAACA/GCCTTCACCTGGTCCCTATC \\
\hline Sugarcane & 12-oxophytodienoate reductase $7, O P R 7$ & TGTTCATCGCTAACCCGGAC/TTAGGCTGGCCAAGGAATGG \\
\hline Sugarcane & Polyadenylate-binding protein 8, PABp8 & TTGGGACTCTGACTTCTGCC/CCAGTGACCTTTGCTGCTTG \\
\hline Sugarcane & Serine/arginine repetitive matrix protein 1, SARMP1 & TGGACTTGGTCAGTTGGAAACA/TGTTCCTGAAGCCTATGTTGCT \\
\hline Sugarcane & Glyceraldehyde-3-phosphate dehydrogenase, GAPDH & AGGACTCCAAGACCCTCCTC/CTTCTTGGCACCACCCTTCA \\
\hline Sugarcane & Eukaryotic elongation factor $1 \mathrm{~A}, e E F 1 A^{a}$ & TाTCACACTTGGAGTGAAGCAGAT/GACTTCCTTCACAATCTCATCATAA \\
\hline Sugarcane & Cullin, $C U L^{\mathrm{a}}$ & TGCTGAATGTGTTGAGCAGC/TTGTCGCGCTCCAAGTAGTC \\
\hline Sugarcane & Clathrin adaptor complex, $C A C^{a}$ & ACAACGTCAGGCAAAGCAAA/AGATCAACTCCACCTCTGCG \\
\hline Sporisorium scitamineum & Conserved hypothetical protein, $\$ 2$ & ACCTCGAGCAGCAACAGTG/ACCACAATCCAGAACTCGACG \\
\hline Sporisorium scitamineum & Conserved hypothetical protein, $\$ 4$ & GACGGTGCCCAAGAACAGAG/CTGTGAGCTTCCAATTCCGC \\
\hline Sporisorium scitamineum & VPS73-protein involved in vacuolar protein sorting, S6 & AAAACCTAATGGTGGGCTCGG/GACCCAACCCGAACGAGAAC \\
\hline Sporisorium scitamineum & Synaptobrevin, S8 & TGCACAAGACCATCGACTCA/CGAGTTTGCTTCTTGGCTGT \\
\hline Sporisorium scitamineum & GTP binding protein Rac1, 59 & CACGTGATATCCATGCGAACAAG/GAGAATGGTGCAGTTGTTCTTCT \\
\hline Sporisorium scitamineum & Inosine 5'-monophosphate dehydrogenase, S10 & CGTTGCAGGACATGGGTGTG/TTCTCGTAGCTGTGCAGACCA \\
\hline Sporisorium scitamineum & SEC65-signal recognition particle subunit, S11 & GAATGCTTGGAGGCATGGGG/GCGGGTTCATAGGGTCCTTC \\
\hline Sporisorium scitamineum & ADP-ribosylation factor, S12 & AACGACCGAGAGCGTGTTTC/AGCTTGTCCGTAATCTCGGC \\
\hline
\end{tabular}

Note: a Ling et al., 2014

cycle threshold $(\mathrm{Ct})$ values of all samples were used to calculate the mean value, standard deviation, and covariation $(\mathrm{CV}, \mathrm{CV}=$ Standard deviation/Average $\mathrm{Ct}$ value $\times 100 \%)$ value. The mean $\mathrm{Ct}$ values were $\Delta \mathrm{Ct}$-transformed and then directly imported into geNorm [26] and NormFinder [27], following the manuals' instructions. Analysis of the stability coefficient of the candidate PCR reference genes on BestKeeper [28] and use of the deltaCt method [8] was as previously described. Pearson correlation coefficients ( $\mathrm{r}$ values) were analyzed with the software SAS S21.0 using the stability values (SV) generated by geNorm, NormFinder, deltaCt, and BestKeeper [24]. The algorithms with generally similar results were used to generate the comprehensive stability values [24]. The relative SVs (Relative $\mathrm{SV}=(\mathrm{SV}$ of rank $\mathrm{N}) /(\mathrm{SV}$ of Rank 1$), N=1 \sim 10)$ were achieved based on the SVs from the algorithms and the deltaCt method. The comprehensive SV (CSV) was computed from the geometrical mean (GM) of the relative SVs of each candidate PCR reference gene. The best combination of PCR reference genes was recommended by the geNorm [26]. To verify the reliability of the selected PCR reference genes, the expression of sugarcane chitinase I-3 (ScChi I-3) [43] and S. scitamineum chorismate mutase (SsCMU) [44] was normalized using the selected candidates, including the best candidate PCR reference gene, the most variable candidate PCR reference gene, and the optimal combination candidate PCR reference genes, respectively. The relative expression of ScChi $I-3$ and $S s C M U$ was calculated with the formula previously described by Pfaffl et al. [45], which are both implemented in OriginPro 9.2.

\section{Results}

Identification of candidate PCR reference genes

Because the CV value is an indicator of the degree of discretization in a group, the lower the $\mathrm{CV}$ value, the less variation among groups. In transcriptomic data shown in Table 2 [3], except for $C A C$, the $C V$ values of $G A P D H, e E F 1 A$, and $C U L$ were $2.52 \%, 12.59 \%$, and $5.22 \%$. The CV values of $A C A D, C K 1 \delta$, OTU5, OPR7, $P A B P 8$, and SARMp1 were between $2.65 \%$ and $3.88 \%$, and their expression was higher than that of GAPDH (Table 2). However, the expression levels of the eight $S$. scitamineum genes, S2, S4, S6, S8, S9, S10, S11, and S12 were also relatively high; however, these showed slight variations $(1.61 \% \sim 4.31 \%)$ in the genomic-wide expression profile microarray (Table 3 ). The above 10 sugarcane genes and eight $S$. scitamineum genes were then selected as candidate PCR reference genes (Table 1) and further evaluated.

\section{Quality evaluation of the primers of the candidate PCR reference genes}

Following the MIQE guidline [46], the melting curve of each the primer pair was analyzed, and the primer pairs of all these 18 candidate PCR reference genes with high amplifying-specificity were selected and used in the subsequent qRT-PCR analysis (Additional file 1: Figure S1 
Table 2 The expression analysis of ten sugarcane candidate PCR reference genes (CRG) based on the RPKM value from RNA-seq data

\begin{tabular}{|c|c|c|c|c|c|c|c|c|c|c|c|}
\hline \multirow[t]{2}{*}{ CRG } & \multicolumn{4}{|l|}{ YC05-179 } & \multicolumn{4}{|l|}{$\mathrm{ROC22}$} & \multirow[t]{2}{*}{ mean } & \multirow[t]{2}{*}{ SD } & \multirow[t]{2}{*}{$C V$} \\
\hline & CK-24 h & $J-24 h$ & $J-48 h$ & $J-120 h$ & CK-24 h & $J-24 h$ & $J-48 h$ & $J-120 h$ & & & \\
\hline$A C A D$ & 37.82 & 37.89 & 34.78 & 37.11 & 37.01 & 34.56 & 35.16 & 36.61 & 36.37 & 1.35 & $3.70 \%$ \\
\hline CK1ठ & 91.73 & 89.52 & 94.76 & 92.68 & 94.37 & 87.24 & 93.32 & 98.05 & 92.71 & 3.32 & $3.58 \%$ \\
\hline OTU5 & 69.95 & 72.46 & 71.13 & 77.11 & 71.21 & 69.04 & 75.94 & 71.98 & 72.35 & 2.81 & $3.88 \%$ \\
\hline OPR7 & 51.73 & 52.14 & 55.65 & 53.10 & 48.94 & 52.02 & 51.81 & 50.56 & 51.99 & 1.93 & $3.72 \%$ \\
\hline PABp8 & 137.92 & 135.44 & 131.73 & 124.45 & 138.25 & 131.10 & 138.93 & 135.35 & 134.15 & 4.87 & $3.63 \%$ \\
\hline SARMp 1 & 31.50 & 32.97 & 31.20 & 32.83 & 32.69 & 31.46 & 31.33 & 30.79 & 31.85 & 0.85 & $2.65 \%$ \\
\hline GAPDH & 30.31 & 30.21 & 29.81 & 29.19 & 28.36 & 28.71 & 30.26 & 29.30 & 29.52 & 0.74 & $2.52 \%$ \\
\hline eEFIA & 2520.172 & 2233.869 & 2788.05 & 1980.246 & 2260.422 & 1920.52 & 2271.531 & 2100.286 & 2259.387 & 284.4435 & $12.59 \%$ \\
\hline CUL & 107.9021 & 105.826 & 101.8729 & 111.1481 & 117.8406 & 113.4682 & 118.8913 & 111.88 & 111.1037 & 5.800734 & $5.22 \%$ \\
\hline CAC & - & - & - & - & - & - & - & - & - & - & - \\
\hline
\end{tabular}

Note: YC05-179 and ROC22, sugarcane genotype YC05-179 and ROC22; CRG candidate PCR reference gene, RPKM reads per kilo bases per million reads, CK/J$24 \mathrm{~h},-48 \mathrm{~h}$ and $-120 \mathrm{~h}$ the data from the control/treated (CK/J) sample for CK/J-24 h, $48 \mathrm{~h}, 120 \mathrm{~h}$ incubation (Que et al. 2014), SD standard deviation, CV covariation, The CAC gene was not found in the RNA-seq data

and Additional file 2: Figure S2). Table 4 shows that the regression coefficients of all the primer pairs range from 0.990 to 0.999 , indicating that the amplification efficiency of these primer pairs is accurate and reasonable. The amplification efficiency of the candidate PCR reference genes was between 0.95 and 1.06, and the amplicon length was between $102 \mathrm{bp}$ and $186 \mathrm{bp}$ (Table 4).

\section{Expression analysis of candidate PCR reference genes}

The expression of 10 sugarcane candidate PCR reference genes in smut spores/water-injected sugarcane $(0 \mathrm{~d}, 3 \mathrm{~d}$, and $7 \mathrm{~d}$ ) and eight $S$. scitamineum candidate PCR reference genes in S. scitamineum spores-injected sugarcane ( $3 \mathrm{~d}$ and $7 \mathrm{~d}$ ) were analyzed by qRT-PCR. The results showed that the $\mathrm{Ct}$ value of the 10 sugarcane candidates were within the range of 22.13-29.30 (Fig. 1a). The PABp8 gene showed the highest expression level, whereas OTU5 exhibited the lowest. The Ct value of the eight $S$. scitamineum candidate PCR reference genes was within the range of 28.94-32.61 (Fig. 1c). S4 showed the highest expression, and S10 exhibited the lowest expression. The CV value of the sugarcane candidates ranged from 2.16 to $3.50 \%$ (Fig. 1b), whereas that of the S. scitamineum candidates ranged from 1.93 to $4.00 \%$ (Fig. 1d). In terms of $C V$ values, $A C A D, C U L$, OTU5, CAC, and $S A R M p 1$ showed less variations than the remaining five sugarcane candidates (GAPDH, eEF1A, CK1 $\delta$, OPR7, and $P A B P 8)$ with smut fungus infection, whereas GAPDH exhibited the highest variability (Fig. 1b). Similarly, the expression of $S 4, S 9, S 11$, and $S 10$ was less variable than the remaining four $S$. scitamineum candidates $(S 2, S 6, S 8$, and S12), and $S 6$ was the most variable $S$. scitamineum candidate PCR reference gene in S. scitamineum-infected sugarcane bud tissues (Fig. 1d).

\section{Stability analysis of candidate PCR reference genes}

The algorithms geNorm, NormFinder, BestKeeper and deltaCt were used to analyze gene stability in sugarcane bud samples based on their Ct values, and the results were assessed using the Pearson correlation coefficient. Table 5 shows that different algorithms could generate significantly consistent results in stability evaluation of candidate PCR reference genes, especially geNorm, NormFinder, and deltaCt in sugarcane candidates and geNorm and NormFinder in S. scitamineum candidates. For its negative correlation with geNorm, NormFinder, and deltaCt, the stability value from BestKeeper was not included in the evaluation of the S. scitamineum candidate PCR reference genes in the present study. Tables 6 and 7 show that the genes with higher stability have the smaller stability values (SVs). In terms of the ranking of the sugarcane candidate PCR reference genes, the results generated using geNorm and NormFinder were nearly the same, and the top seven genes were $A C A D>S A R M p 1>$ $C K 1 \delta>C A C>O T U 5>P A B P 8>C U L$. The stability order (from stable to unstable) of the sugarcane candidate PCR reference genes derived from BestKeeper was $S A R M p 1$, ACAD, CAC, OTU5, CUL, PABP8, eEF1A, GAPDH, CK1 $\delta$, and OPR7 (Table 6). Except for the order of CAC/CUL and $C K 1 \delta / G A P D H$ being reverse, the rank of candidates generated by BestKeeper and deltaCt was generally similar. The ranking order of the S. scitamineum candidate PCR reference genes from geNorm and NormFinder was also the same. Among the eight $S$. scitamineum candidates, $S 10$ and S11 were the two most stable genes, whereas $S 6$ was the most variable (Table 7 ). DeltaCt analysis showed that the stability of $S 9$ was higher than the remaining seven $S$. scitamineum candidate PCR reference genes, followed by $S 10, S 12$, and $S 8$ (Table 7), whereas $S 2$ was the most unstable (Table 7). 
Huang et al. BMC Genomics (2018) 19:479

Page 6 of 13

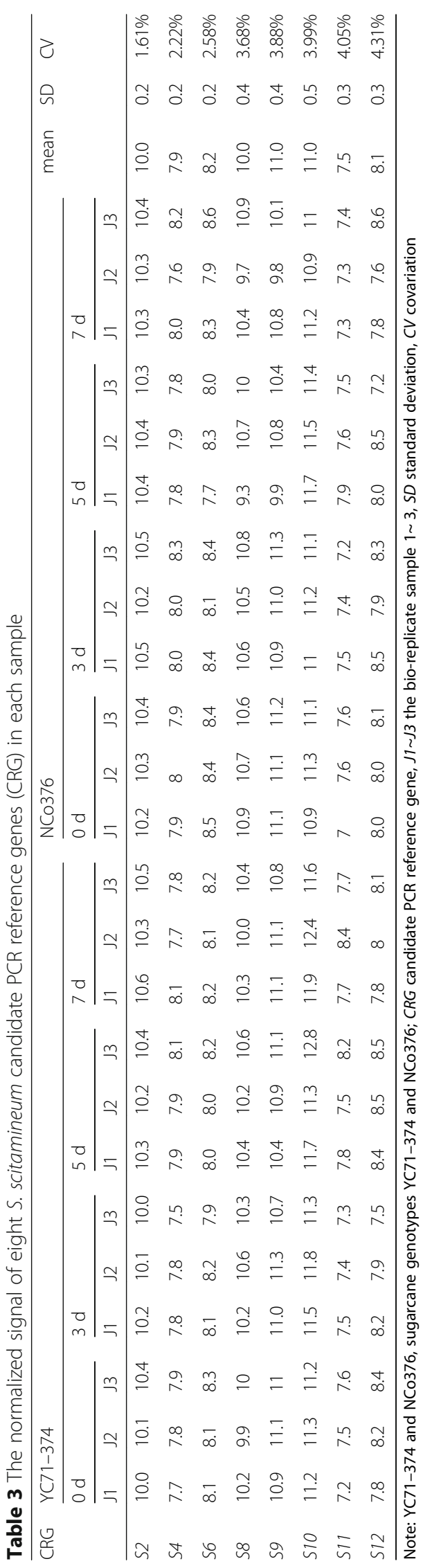


Table 4 The length of qRT-PCR amplicon and the PCR efficiency of 18 candidates PCR reference genes (CRG)

\begin{tabular}{lllll}
\hline Category & CRG & Amplicon length (bp) & $\mathrm{E}(\%)$ & $\mathrm{R}^{2}$ \\
\hline Sugarcane & ACAD & 120 & 1.01 & 0.995 \\
Sugarcane & CK1S & 153 & 1.01 & 0.993 \\
Sugarcane & OTU5 & 186 & 0.99 & 0.992 \\
Sugarcane & OPR7 & 133 & 1.05 & 0.992 \\
Sugarcane & PABP8 & 121 & 1.03 & 0.993 \\
Sugarcane & SARMp1 & 102 & 1.01 & 0.999 \\
Sugarcane & GAPDH & 170 & 1.04 & 0.990 \\
Sugarcane & EEF1A & 103 & 0.97 & 0.999 \\
Sugarcane & CUL & 105 & 1.06 & 0.999 \\
Sugarcane & CAC & 112 & 1.00 & 0.999 \\
Sporisorium scitamineum & S2 & 118 & 1.04 & 0.999 \\
Sporisorium scitamineum & S4 & 130 & 0.99 & 0.990 \\
Sporisorium scitamineum & S6 & 138 & 0.96 & 0.995 \\
Sporisorium scitamineum & S8 & 122 & 0.97 & 0.992 \\
Sporisorium scitamineum & S9 & 143 & 1.04 & 0.993 \\
Sporisorium scitamineum & S10 & 133 & 1.00 & 0.993 \\
Sporisorium scitamineum & S11 & 102 & 1.00 & 0.995 \\
Sporisorium scitamineum & S12 & 146 & 0.97 & 0.996 \\
\hline
\end{tabular}

Note: ${ }^{a}$, Ling et al. 2014
Comprehensive analysis using geNorm, NormFinder, BestKeeper, and deltaCt indicated that the two most stable sugarcane candidate PCR reference genes were $A C A D$ and $S A R M p 1$, followed by $C A C$, whereas the most variable reference gene was GAPDH. Similarly, based on the results of geNorm, NormFinder, and deltaCt, $S 10$ and $S 11$ are the two most stable S. scitamineum candidate PCR reference genes, followed by $S 4$, and $S 6$ is the most unstable.

\section{Selection of the optimal combination of PCR reference genes}

Concurrently, the pairwise variation $(\mathrm{Vn} / \mathrm{Vn}+1)$ of candidate PCR reference gene combination was analyzed using geNorm, which based on the normalized $\mathrm{Ct}$ value [26]. The pairwise variations could reflect the variations among different gene groups, which comprised various genes. Vandesompele et al. [25] emphasized that if the pairwise variation is $<0.15$, then the combination of the top $n$ genes was more stable than the combination of top $n+1$ genes [26]. Figure 2 shows that all pairwise variations of sugarcane and S. scitamineum genes are $<0.15$. Comparisons of the pairwise variations between different gene combinations indicated minimal differences among the $\mathrm{V} 2 / 3, \mathrm{~V} 3 / 4$, and $\mathrm{V} 4 / 5$ of the sugarcane candidate PCR reference genes, and V2/3 was the smallest among all combinations of S. scitamineum candidate PCR reference genes. As using fewer PCR reference genes to achieve the most
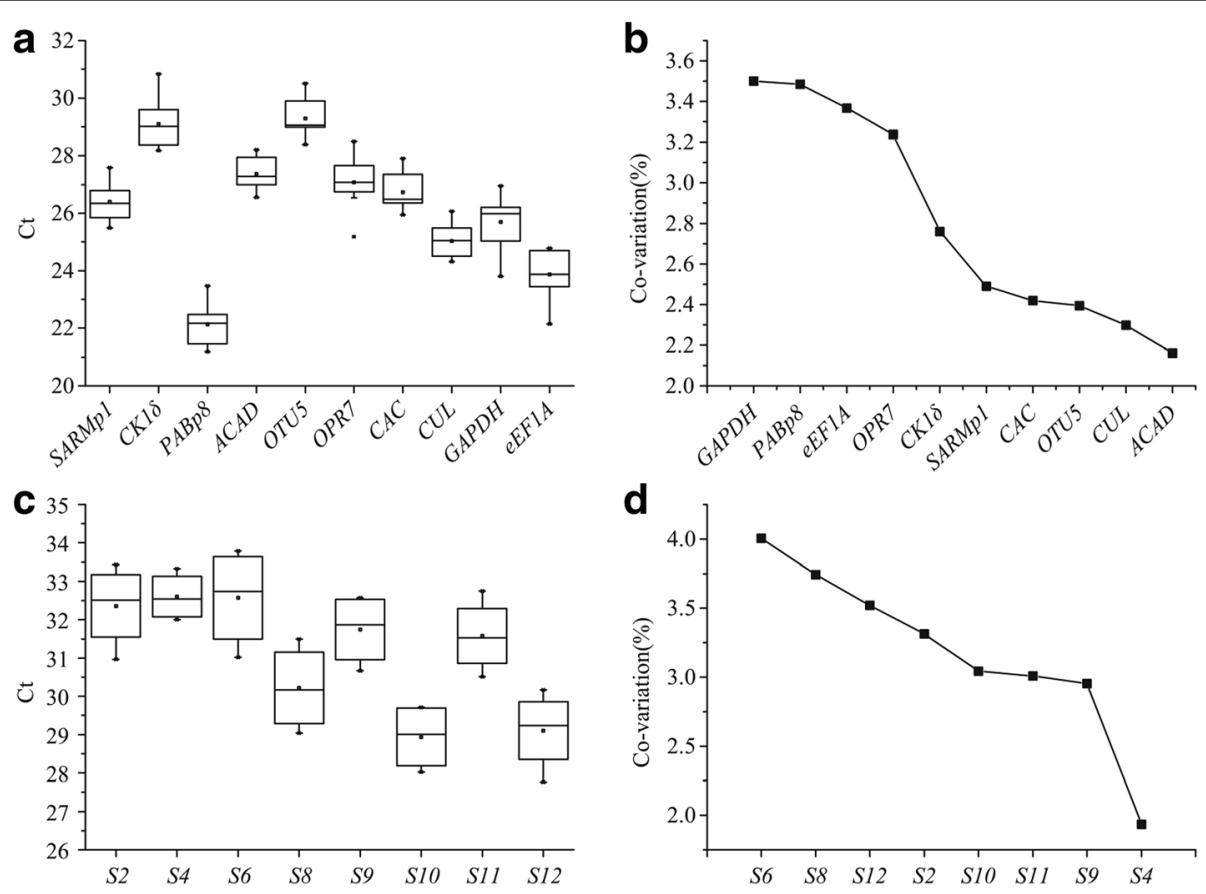

Fig. 1 Cycle threshold (Ct) values and variations in the expression of candidate PCR reference genes. a, the mean Ct value of sugarcane candidate PCR reference genes; $\mathbf{b}$, the expression covariation (CV) of sugarcane candidate PCR reference genes; $\mathbf{c}$, the mean $\mathrm{Ct}$ value of S. scitamineum candidate PCR reference genes; $\mathbf{d}$, the expression $C V$ of $C t$ value of $S$. scitamineum candidate PCR reference genes 
Table 5 Correlation of the stability value of reference gene based on four statistical algorithms

\begin{tabular}{lll}
\hline Algorithms & Correlation & \\
\cline { 2 - 3 } & Sugarcane & Sporisorium scitamineum \\
\hline geNorm vs. NormFinder & $0.964^{* *}$ & $0.993^{* *}$ \\
geNorm vs. deltaCt & $0.694^{*}$ & 0.376 \\
geNorm vs. BestKeeper & 0.594 & -0.696 \\
NormFinder vs. deltaCt & 0.595 & 0.303 \\
NormFinder vs. BestKeeper & 0.425 & -0.700 \\
deltaCt vs. BestKeeper & $0.927^{* *}$ & -0.067 \\
\hline
\end{tabular}

Note: ${ }^{*}$, significant difference $(p<0.05) ;{ }^{* *}$, significant difference $(p<0.01)$

reasonable results is cost-effective, "ACAD + SARMp1" and "S10 + S11" are regarded as the best combinations of sugarcane and $S$. scitamineum candidate PCR reference genes, respectively.

\section{Validation analysis of PCR reference genes}

Based on the results of our analyses, the top two stable sugarcane/S. scitamineum reference genes $(A C A D$ and $S A R M p 1 / S 10$ and $S 11)$, a moderately stable gene (CAC/ $S 4)$, the most variable candidate PCR reference gene (GAPDH/S6), and the optimal combination of PCR reference genes $(A C A D+S A R M p 1 / S 10+S 11)$ were selected for further validation. Here, sugarcane ScChi I-3 gene and $S$. scitamineum $S s C M U$ gene were used to verify the reasonability and feasibility of the above selected genes.

Figure 3a shows that the ScChi I-3 gene was upregulated and exhibited a similar expression pattern when it was normalized with $A C A D, S A R M p 1$, and " $A C A D+$ SARMp1" in the S. scitamineum-infected buds tissue samples of NCo376. The expression level of ScChi I-3, which was normalized with GAPDH, was also upregulated but was higher than that of $A C A D, S A R M p 1$, and "ACAD + SARMp1", respectively. On the contrary, the expression of ScChi I-3 was downregulated when normalized with $C A C$. Similarly, the normalization of ScChi I-3 expression with reference gene "ACAD+ SARMp1", ACAD, SARMp1, and GAPDH was similar in the infected buds tissues of YC71-374 (Fig. 3b), whereas the $S c C h i$ I-3 expression level with $C A C$ as reference was relatively higher.

The expression level of the $S s C M U$ gene could not be detected in S. scitamineum-infected sugarcane buds at 0 d. Figure 4 ( $0 \mathrm{~d}$ was excluded in the analysis) shows that the expression of SsCMU gradually increased with $S$. scitamineum infestation in sugarcane tissues when the data were normalized using the two best candidate PCR reference genes (S10 and S11) and their combination. Otherwise, $S s C M U$ expression with $S 4$ or $S 6$ normalization was higher and differed from that with normalization using S10, $S 11$, or their combination $(p<0.05)$ (Fig. 4). Moreover, the expression of $S s C M U$ among the three bio-replicates, which was normalized with $S 4$ or $S 6$, was relatively more variable (with greater ranges) (Fig. 4).

\section{Discussion}

Quantification of defense response-related gene expression is an important method for elucidating the molecular mechanisms of plant-pathogen and plant-environmental factor interactions [47]. Compared to Northern blotting, ribonuclease protection assay (RPA), semi-qPCR, molecular in situ hybridization, and cDNA microarray, the combination of qRT-PCR and data normalization using PCR reference genes is a more rapid, convenient, and reliable way of assessing gene transcriptional levels $[46,48]$. It has eventually become one of the most indispensable means for assessing gene expression in animals and plants [49]. The ideal reference gene should have stable expression levels in bio-samples, encodes a functional protein, and is transcriptionally abundant (with a $\mathrm{Ct}$ ranged within 15 to 30) [50]. It should also reflect variations in RNA extraction, cDNA synthesis, and PCR amplification [50]. Ten

Table 6 The relative stability value (RSV) of ten sugarcane candidate PCR reference genes

\begin{tabular}{|c|c|c|c|c|c|c|c|c|c|c|}
\hline & \multicolumn{2}{|l|}{ geNorm } & \multicolumn{2}{|c|}{ NormFinder } & \multicolumn{2}{|c|}{ BestKeeper } & \multicolumn{2}{|l|}{ DeltaCt } & \multicolumn{2}{|c|}{ Comp. Rank } \\
\hline & Gene & RSV & Gene & RSV & Gene & RSV & Gene & RSV & Gene & CSV \\
\hline 1 & $A C A D$ & 1.00 & $A C A D$ & 1.00 & SARMpl & 1.00 & SARMP1 & 1.00 & $A C A D$ & 1.01 \\
\hline 2 & SARMP1 & 1.00 & SARMP1 & 1.08 & $A C A D$ & 1.02 & $A C A D$ & 1.01 & SARMp 1 & 1.02 \\
\hline 3 & CK1ס & 1.74 & $C K 1 \delta$ & 2.00 & $C A C$ & 1.03 & CUL & 1.06 & $C A C$ & 1.53 \\
\hline 4 & $C A C$ & 2.13 & $C A C$ & 2.36 & OTU5 & 1.05 & OTU5 & 1.06 & OTU5 & 1.69 \\
\hline 5 & OTU5 & 2.53 & OTU5 & 2.89 & CUL & 1.05 & $C A C$ & 1.06 & $C K 1 \delta$ & 1.86 \\
\hline 6 & PABP8 & 3.41 & PABP8 & 3.14 & PABP8 & 1.08 & PABP8 & 1.21 & PABP8 & 1.93 \\
\hline 7 & CUL & 4.54 & CUL & 4.11 & $e E F 1 A$ & 1.23 & eEF1A & 1.35 & CUL & 2.13 \\
\hline 8 & eEF1A & 5.49 & OPR7 & 5.13 & GAPDH & 1.67 & CK1ठ & 1.68 & eEF1A & 2.66 \\
\hline 9 & GAPDH & 6.52 & eEF1A & 5.48 & CK1ठ & 2.04 & GAPDH & 1.70 & GAPDH & 3.23 \\
\hline 10 & OPR7 & 7.31 & GAPDH & 5.85 & OPR7 & 3.24 & OPR7 & 2.02 & OPR7 & 3.96 \\
\hline
\end{tabular}

Note: SV stability value, Comp. Rank comprehensive rank, RSV relative SV, CSV comprehensive SV 
Table 7 The relative stability value (RSV) of S. scitamineum candidate PCR reference genes

\begin{tabular}{|c|c|c|c|c|c|c|c|c|}
\hline & \multicolumn{2}{|c|}{ geNorm } & \multicolumn{2}{|c|}{ NormFinder } & \multicolumn{2}{|c|}{ DeltaCt } & \multicolumn{2}{|c|}{ Comp. Rank } \\
\hline & Gene & RSV & Gene & RSV & Gene & RSV & Gene & CSV \\
\hline 1 & $S 10$ & 1.00 & $S 10$ & 1.00 & 59 & 1.00 & 510 & 1.01 \\
\hline 2 & S11 & 1.00 & $S 11$ & 1.00 & $\$ 10$ & 1.02 & $S 11$ & 1.12 \\
\hline 3 & S4 & 2.69 & S4 & 4.96 & $S 12$ & 1.39 & S4 & 2.68 \\
\hline 4 & $S 12$ & 15.34 & $S 12$ & 53.21 & 58 & 1.40 & $S 12$ & 10.43 \\
\hline 5 & S8 & 21.48 & S8 & 98.47 & $S 11$ & 1.41 & 58 & 14.36 \\
\hline 6 & 59 & 31.63 & 59 & 166.06 & S4 & 1.45 & 59 & 17.38 \\
\hline 7 & S2 & 37.00 & S2 & 169.24 & S6 & 1.47 & $S 2$ & 23.71 \\
\hline 8 & 56 & 44.43 & 56 & 231.21 & S2 & 2.13 & 56 & 24.72 \\
\hline
\end{tabular}

Note: SV stability value, Comp. Rank comprehensive rank, RSV relative SV, CSV comprehensive SV

sugarcane genes (GAPDH, eEF1A, CUL, CAC, ACAD, CK1 $\delta$, OTU5, OPR7, PABP8, and SARMp1) and eight $S$. scitamineum genes (S2, S4, S6, S8, S9, S10, S11, and S12) were selected as candidate PCR reference genes for stability evaluation. According to the MIQE criteria [46], qRT-PCR primer pairs with amplification efficiency within the range of 0.95-1.05 were selected for quantitative expression profiling. Pearson correlation analysis showed that two algorithms, namely, geNorm and NormFinder, and the deltaCt method shared more similar ranking results candidate in PCR reference genes, whereas less similar findings were generated between BestKeeper and the algorithms geNorm, NormFinder or the deltaCt method, especially in the smut candidate PCR reference genes. Finally, three algorithms, namely, geNorm, NormFinder BestKeeper, and the deltaCt method were used in the analysis of sugarcane candidate PCR reference genes, while two algorithms, geNorm, NormFinder, and deltaCt method were used in the analysis of smut candidate PCR reference genes.

\section{Selection of sugarcane PCR reference genes}

On the basis of reference gene selection criteria [50], the $\mathrm{Ct}$ values of the 18 candidate PCR reference genes were within the range 15-30, except for CK1 $\delta$ and OTU5 (Ct value $>30$ ) (Fig. 1a). Previous studies have shown that the sugarcane GAPDH and $e E F 1 A$ genes were stable and were deemed suitable for use as PCR reference genes under osmotic, drought, or hormonal stress [23-25], but were variable during S. scitamineum infection in sugarcane buds in the present study (Fig. 1b). Similarly, the expression of $G A P D H$ in plant tissues varied under biotic stress such as in virus-infected $N$. benthamiana [51], rust-infected Vigna angularis [52], and fungi-infected Withania somnifera [53]. Xiao et al. [50] reported that the expression level of $e E F 1 A$ in Chinese cabbage was altered by fungal infection [54]. This indicates that the expression of GAPDH and $e E F 1 A$ in plants is sensitive to biotic stress. The transcriptomic data and the present data from geNorm, NormFinder, BestKeeper, and deltaCt method indicated that $A C A D$ and $S A R M p 1$ are the two most stable genes among the 10 sugarcane candidates. The ACAD protein is involved in the electron transport chain and is one of the most basic enzymatic components in peroxisomes, which is a basic organelle of eukaryotic cells [55]. The findings of the present study suggest that $A C A D$ is suitable for use as a reference gene for normalization of data on differentially expressed genes that are related to sugarcane- $S$. scitamineum interactions, although $A C A D$ has not been employed as a reference gene prior to this study. Similarly, the SARMp1 protein is a member of serine/ arginine-rich protein family, which participates in the splicing of mRNA precursors [56, 57]. Compared to GAPDH, EF1A, CAC, and CUL [23, 24], both ACAD and $S A R M p 1$ are the basic components for cellular structures and may also serve as PCR reference genes in sugarcane. Several previous studies have proven that
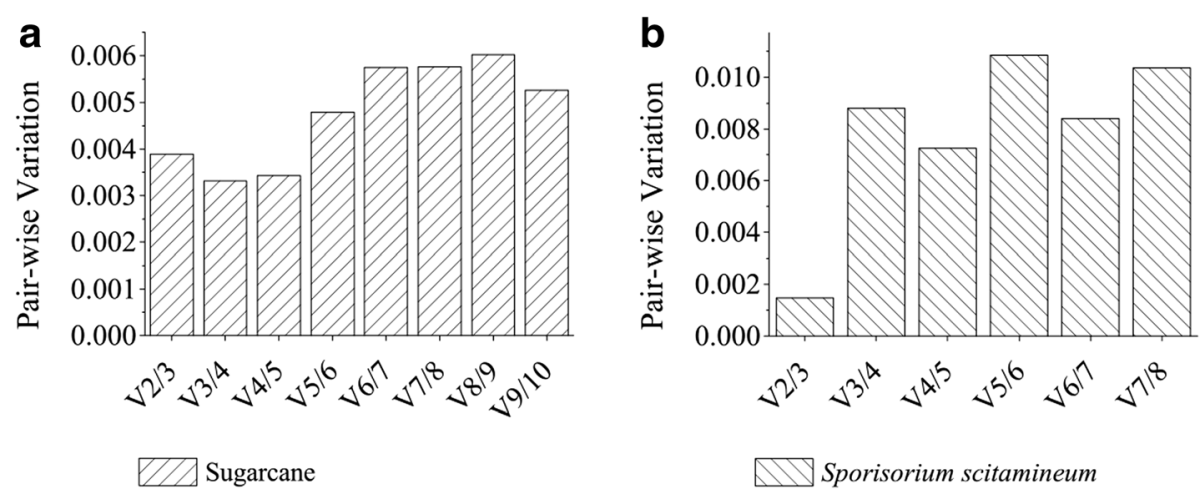

Fig. 2 Determination of the optimal number of sugarcane (a) and S. scitamineum (b) reference genes for normalization by pairwise variation. Pairwise variation $(\mathrm{Vn} N \mathrm{n}+1)$ was analyzed between normalization factors $\mathrm{NFn}$ and $\mathrm{NFn}+1$ by geNorm algorithm to determine $(\mathrm{V}<0.15)$ the optimal number of reference genes 

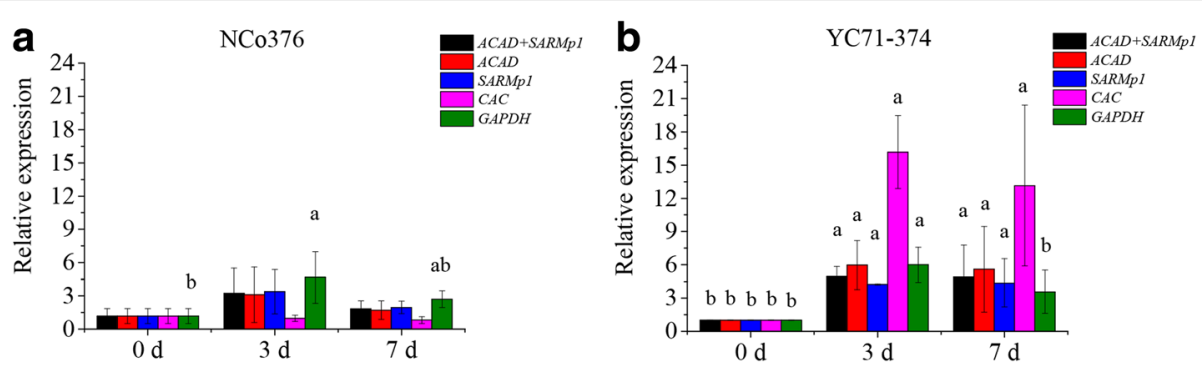

Fig. 3 The relative expression of sugarcane chitinase I-3 gene in NCo376 (a) and YC71-374 (b) bud tissue under S. scitamineum infection. The $\mathrm{a}$ and $\mathrm{b}$ represent significant differences between the normalization of different reference genes or gene combinations

the gene combination recommended by geNorm is the most suitable internal reference for gene expression [58-60]. In the present study, the results from geNorm support that $A C A D$ and $S A R M p 1$ could be used as a combination for normalization of data on gene expression.

Based on all the above results, the top two stable genes $A C A D$ and $S A R M p 1$ and the moderately stable gene $C A C$ plus the unstable gene GAPDH were selected for further validation in S. scitamineum-infected NCo376 and YC71-374 bud samples. The results finally showed that the transcript profile of ScChi I-3 that was normalized using $C A C$ and GAPDH was significantly different from those by $A C A D, S A R M p 1$ and "ACAD + SARMp1", and was more obviously changeable. In addition, $A C A D$ and "ACAD+SARMp1" generated the most similar normalization results in both smut-susceptible and -resistant genotypes in the present study. In sum, based on the evaluation of four algorithms and qRT-PCR validation, $A C A D$ or "ACAD + SARMp1" are deemed the most suitable PCR reference genes for transcriptional quantification in S. scitamineum-infected sugarcane buds.

\section{Selection of S. scitamineum PCR reference genes}

With the completion of the $S$. scitamineum genome sequencing, nearly 6636-6693 genes have been identified, which consist of 527 secreted protein genes, 192 pathogenic genes, and 68 effector proteins [35-37]. Some $S$. scitamineum-specific genes are expressed as part of the immune response of sugarcane cells to infection [36]. Prior to the identification of the function of these pathogenesis-related $S$. scitamineum genes, it is essential to know which gene is expressed differentially during infection. Internal PCR reference genes could enhance the reliability of identification of differentially expressed genes using qRT-PCR [3]. However, no report on the appropriate $S$. scitamineum PCR reference genes has been published to date.

Based on the data from a genomic-wide expression profile microarray (unpublished), eight S. scitamineum candidate PCR reference genes were selected for further stability evaluation using qRT-PCR and different algorithms. The results showed that the accumulation of $S 10$ (mean $\mathrm{Ct}=28.94)$ and $\mathrm{S12}$ (mean $\mathrm{Ct}=29.10$ ) was within the $\mathrm{Ct}$ range of 15 to 30 . During the detection of the S. scitamineum gene in sugarcane bud tissues, the transcriptional abundance of S. scitamineum genes is predictably lower than that of host genes, resulting in the $\mathrm{Ct}$ value of six S. scitamineum genes $>30 \mathrm{Ct}$. In other words, if the $\mathrm{Ct}$ value of the pathogen gene is $>30$, then it does not mean that the expression of the S. scitamineum gene is low. Vieira et al. found that the Ct values of the Colletotrichum kahawae genes in the tissues of Arabica coffee hypocotyls were higher than in the medium [48]. Therefore, $S 2, S 4$, $S 6, S 8, S 9$, and $S 11$ in this study could also be considered as appropriate PCR reference genes. Based on the CV values of eight $S$. scitamineum genes, the expression of $S 4$,
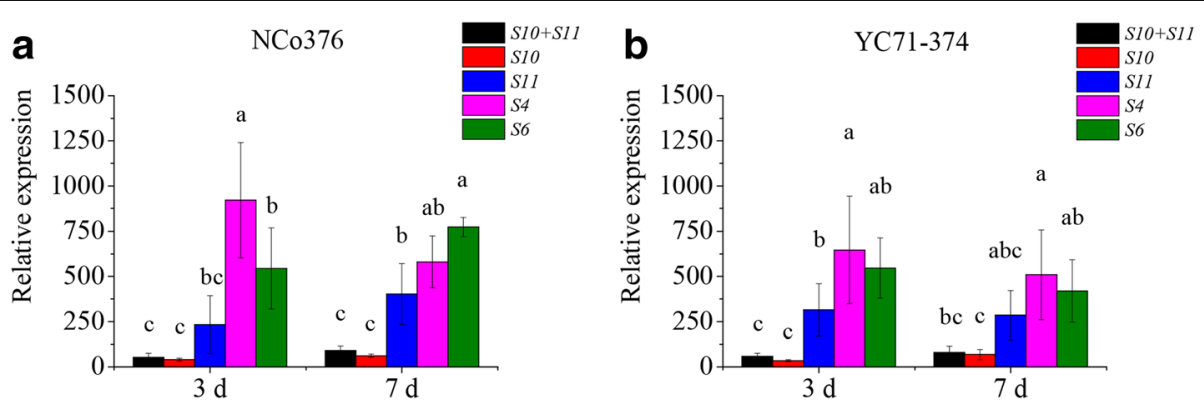

Fig. 4 The relative expression of S. scitamineum chorismate mutase gene in S. scitamineum-infested sugarcane NCo376 (a) and YC71-374 (b) bud tissues. $a$, b, and c represent significant differences in normalization of different reference genes or gene combinations 
$S 9, S 10$, and $S 11$ is less variable, whereas that of $S 6$ is the most variable. Based on the results using geNorm, NormFinder, and deltaCt method, S10 and S11 are more transcriptionally stable than $S 4$ and $S 9$. However, the results of $\mathrm{CV}$ value analysis and geNorm, NormFinder, and deltaCt all indicated that $S 6$ is the most transcriptionally unstable gene. The $S 10$ gene encodes inosine-5-monophosphate dehydrogenase, which is a rate-limiting enzyme that regulates intracellular nucleic acids levels in the guanine de novo synthesis pathway [61, 62]. The $S 11$ gene encodes one of the key components of the signal recognition complex [63]. These features suggest that both $S 10$ and $S 11$ are indispensable proteins in living cells and thus are considered as housekeeping genes. In the same samples, the expression of $S s C M U$ under the normalization of $S 4$ and $S 6$ were significantly higher $(p<0.05)$ than that of $S 10$, S11, and their combination, indicating that the unsuitable reference gene may generate unreliable transcript profiles of the target gene. The results of the present study have proven that $S 10, S 11$, or their combination may be utilized as a reliable tool for normalization of expression data on S. scitamineum genes in the sugarcane buds.

\section{Conclusions}

In this study, 10 sugarcane genes and eight S. scitamineum genes were selected as the candidate PCR reference genes based on the reported sugarcane transcriptome during $S$. scitamineum infection and the sugarcane-S. scitamineum expression microarray data. To identify the stability and the best combination and applicability of the 18 candidate PCR reference genes, they were quantitatively analyzed in sugarcane buds of different varieties and infection times by qRT-PCR. ACAD, SARMp1 and "ACAD + SARMp1" were identified as optimal sugarcane reference genes/gene combination, and S10, S11, and "S10 + S11" were identified as optimal S. scitamineum reference genes/gene combination. This study facilitates gene expression analysis in sugarcane-S. scitamineum interaction systems.

\section{Additional files}

Additional file 1: Figure S1. Melting curves of sugarcane candidate PCR reference genes. (TIF $3140 \mathrm{~kb}$ )

Additional file 2: Figure S2. Melting curves of S. scitamineum candidate PCR reference genes. (TIF $10506 \mathrm{~kb}$ )

\footnotetext{
Abbreviations

ACAD: Acyl-CoA dehydrogenase; CAC: Clathrin adaptor complex; CK18: Casein kinase I isoform delta-like; CRG: Candidate PCR reference genes; Ct: Cycle threshold; CUL: Cullin; CV: Covariation; eEF1A: Eukaryotic elongation factor 1A; GAPDH: Glyceraldehyde-3-phosphate dehydrogenase; OPR7: 12-

Oxophytodienoate reductase 7; OTU5: OTU domain-containing protein 5; PABP8: Polyadenylate-binding protein 8; qRT-PCR: Real-time quantitative PCR; RPA: Ribonuclease protection assay; RPKM: Reads per kilo bases per million reads; S. scitamineum: Sporisorium scitamineum; SARMP 1: Serine/arginine repetitive matrix protein 1 gene; ScChi I-3: Sugarcane chitinase I-3;
}

SD: Standard deviation; semi-qPCR: Semi-quantitative PCR; SSCMU: S. scitamineum chorismate mutase; SV: Stability value

\section{Acknowledgments}

We sincerely thank Qinnan Wang (Hainan Sugarcane Breeding Station of Guangzhou Sugarcane Industry Research Institute) for providing primary seed-canes of the sugarcane genotypes NCo376 and YC71-374, as well as Dinggang Zhou in our lab for assisting in the RNA isolations.

\section{Funding}

The National Natural Science Foundation of China (31671752, 31101196, and 31340060), Natural Science Foundation of Fujian Province, China (2015 J06006), the earmarked fund for the Modern Agriculture Technology of China (CARS-17), the Program for New Century Excellent Talents in Fujian Province University (JA14095) supported this study, and Special fund for science and technology innovation of Fujian Agriculture And Forestry University (KFA17267A). These funding agencies had no role in the design of the study and collection, analysis, and interpretation of data and in writing the manuscript.

\section{Availability of data and materials}

The data supporting the conclusions of this article are within the paper.

\section{Authors' contributions}

YXQ initiated the project. $Y X Q, N H, H L$, and RKC conceived and designed the experiments. $\mathrm{NH}, \mathrm{HL}$, and $\mathrm{FL}$ prepared the materials and reagents. $\mathrm{NH}, \mathrm{HL}, \mathrm{FL}$, WHS, HYM, XZ, and LW performed the experiments. YXQ, NH, HL, and YCS analyzed the data. $\mathrm{NH}$ and $\mathrm{HL}$ prepared the tables and drafts. $\mathrm{NH}$ and $\mathrm{HL}$ written the original draft. YXQ, NH, HL and YCS reviewed and edited the manuscript. All authors have read and approved the manuscript.

\section{Ethics approval and consent to participate}

We confirm that no specific permits were required for the described locations/activities. We also confirm that the field studies did not involve endangered or protected species.

\section{Competing interests}

The authors declare that they have no competing interests.

\section{Publisher's Note}

Springer Nature remains neutral with regard to jurisdictional claims in published maps and institutional affiliations.

\section{Author details}

${ }^{1}$ Key Laboratory of Sugarcane Biology and Genetic Breeding, Ministry of Agriculture, Fujian Agriculture and Forestry University, Fuzhou 350002, China. ${ }^{2}$ Key Laboratory of Ministry of Education for Genetics, Breeding and Multiple Utilization of Crops, College of Crop Science, Fujian Agriculture and Forestry University, Fuzhou 350002, China. ${ }^{3}$ Guangxi Collaborative Innovation Center of Sugarcane Industry, Guangxi University, Nanning 530005, China.

Received: 6 September 2017 Accepted: 6 June 2018

Published online: 19 June 2018

\section{References}

1. Manners JM. Functional genomics of sugarcane. Adv Bot Res. 2011;60:89-168.

2. Vieira A, Talhinhas P, Loureiro A, Duplessis S, Fernandez D, Silva MC, Paulo OS, Azinheira HG. Validation of RT-qPCR reference genes for in planta expression studies in Hemileia vastatrix, the causal agent of coffee leaf rust. Fungal Biol. 2011;115:891-901.

3. Que Y, Su Y, Guo J, Wu Q, Xu L. A global view of transcriptome dynamics during Sporisorium scitamineum challenge in sugarcane by RNA-Seq. PLoS One. 2014;9:e106476.

4. Wu Q, Xu L, Guo J, Su Y, Que Y. Transcriptome profile analysis of sugarcane responses to Sporisorium scitaminea infection using Solexa sequencing technology. Biomed Res Int. 2013;2013:298920.

5. Kumar A, Naik GK, Giridhar P. Dataset on exogenous application of salicylic acid and methyljasmonate and the accumulation of caffeine in young leaf tissues and catabolically inactive endosperms. Data Brief. 2017;13:22-7.

6. Fan W, Chang W, Liu X, Xiao C, Yang J, Zhang Z. Identification of up-regulated genes provides integrated insight into salt-induced tolerance mechanisms in Sesuvium portulacastrum roots. Acta Physiol Plant. 2017;39:86. 
7. Pfaffl MW. Relative quantification. Real-time PCR. 2006;63:63-82.

8. Silver N, Best S, Jiang J, Thein SL. Selection of housekeeping genes for gene expression studies in human reticulocytes using real-time PCR. BMC Mol Biol. 2006; 7:33.

9. Thellin O, Zorzi W, Lakaye B, De Borman B, Coumans B, Hennen G, Grisar T, Igout A, Heinen E. Housekeeping genes as internal standards: use and limits. J Biotechnol. 1999;75:291-5.

10. Gutierrez L, Mauriat M, Pelloux J, Bellini C, van Wuytswinkel O. Towards a systematic validation of references in real-time RT-PCR. Plant Cell. 2008;20:1734-5.

11. Sun Z-B, Li S-D, Sun M-H. Selection of reliable reference genes for gene expression studies in Clonostachys rosea 67-1 under sclerotial induction. J Microbiol Meth. 2015;114:62-5.

12. Chen C, Xie T, Ye S, Jensen AB, Eilenberg J. Selection of reference genes for expression analysis in the entomophthoralean fungus Pandora neoaphidis. Braz J Microbiol. 2016;47:259-65.

13. Volkov RA, Panchuk II, Schoffl F. Heat-stress-dependency and developmental modulation of gene expression: the potential of house-keeping genes as internal standards in mRNA expression profiling using real-time RT-PCR. J Exp Bot. 2003;54:2343-9.

14. Nicot N, Hausman JF, Hoffmann L, Evers D. Housekeeping gene selection for real-time RT-PCR normalization in potato during biotic and abiotic stress. J Exp Bot. 2005;56:2907-14.

15. Lilly S, Drummond R, Pearson M, MacDiarmid R. Identification and validation of reference genes for normalization of transcripts from virus-infected Arabidopsis thaliana. Mol Plant-Microbe Interact. 2011;24:294-304.

16. Jain M, Nijhawan A, Tyagi AK, Khurana JP. Validation of housekeeping genes as internal control for studying gene expression in rice by quantitative realtime PCR. Biochem Biophys Res Commun. 2006;345:646-51.

17. Manoli A, Sturaro A, Trevisan S, Quaggiotti S, Nonis A. Evaluation of candidate reference genes for qPCR in maize. J Plant Physiol. 2012;169:807-15.

18. Chandna R, Augustine R, Bisht NC. Evaluation of candidate reference genes for gene expression normalization in Brassica juncea using real time quantitative RT-PCR. PLoS One. 2012;7:e36918.

19. Paolacci AR, Tanzarella OA, Porceddu E, Ciaffi M. Identification and validation of reference genes for quantitative RT-PCR normalization in wheat. BMC Mol Biol. 2009:10:11.

20. Cortleven A, Remans T, Brenner WG, Valcke R. Selection of plastid- and nuclear-encoded reference genes to study the effect of altered endogenous cytokinin content on photosynthesis genes in Nicotiana tabacum. Photosynth Res. 2009:102:21-9.

21. Mehnaz S. Microbes - friends and foes of sugarcane. J Basic Microb. 2013;53: 954-71.

22. Iskandar HM, Simpson RS, Casu RE, Bonnett GD, Maclean DJ, Manners $J M$. Comparison of reference genes for quantitative real-time polymerase chain reaction analysis of gene expression. Plant Mol Biol Rep. 2004;22:325-37.

23. Ling H, Wu Q, Guo J, Xu L, Que Y. Comprehensive selection of reference genes for gene expression normalization in sugarcane by real time quantitative RT-PCR. PLoS One. 2014;9:e97469.

24. Guo J, Ling $H, W u Q, X u L$, Que $Y$. The choice of reference genes for assessing gene expression in sugarcane under salinity and drought stresses. Sci Rep. 2014;4:7042.

25. de Oliveira Silva RL, Silva MD, Costa Ferreira Neto JR, de Nardi CH, Chabregas SM, Burnquist WL, Kahl G, Benko-Iseppon AM, Kido EA. Validation of novel reference genes for reverse transcription quantitative real-time PCR in drought-stressed sugarcane. Scientific World J. 2014;12:357052.

26. Vandesompele J, De Preter K, Pattyn F, Poppe B, Van Roy N, De Paepe A, Speleman F. Accurate normalization of real-time quantitative RT-PCR data by geometric averaging of multiple internal control genes. Genome Biol. 2002;3 research0034.1.

27. Andersen $\mathrm{CL}$, Jensen $\mathrm{J}$, Orntoft TF. Normalization of real-time quantitative reverse transcription-PCR data: a model-based variance estimation approach to identify genes suited for normalization, applied to bladder and colon cancer data sets. Cancer Res. 2004:64:5245-50.

28. Pfaffl MW, Tichopad A, Prgomet C, Neuvians TP. Determination of stable housekeeping genes, differentially regulated target genes and sample integrity: BestKeeper - excel-based tool using pair-wise correlations. Biotechnol Lett. 2004;26:509-15.

29. He Y, Yan H, Hua W, Huang Y, Wang Z. Selection and validation of reference genes for quantitative real-time PCR in gentiana macrophylla. Front Plant Sci. 2016;7:945
30. Reddy DS, Bhatnagar-Mathur P, Reddy PS, Cindhuri KS, Ganesh AS, Sharma KK. Identification and validation of reference genes and their impact on normalized gene expression studies across cultivated and wild cicer species. PLoS One. 2016;11:e0148451.

31. Borges AF, Fonseca C, Ferreira RB, Lourenco AM, Monteiro S. Reference gene validation for quantitative RT-PCR during biotic and abiotic stresses in vitis vinifera. PLoS One. 2014;9:e111399.

32. Machado RD, Christoff AP, Loss-Morais G, Margis-Pinheiro M, Margis $R$, Koerbes AP. Comprehensive selection of reference genes for quantitative gene expression analysis during seed development in Brassica napus. Plant Cell Rep. 2015;34:1139-49.

33. Huang D-L, Gao Y-J, Gui Y-Y, Chen Z-L, Qin C-X, Wang M, Liao Q, Yang L-T, Li Y-R. Transcriptome of high-sucrose sugarcane variety GT35. Sugar Tech. 2016;18:520-8.

34. Vicentini R, Felix JD, Dornelas MC, Menossi M. Characterization of a sugarcane (Saccharum spp.) gene homolog to the brassinosteroid insensitive1-associated receptor kinase 1 that is associated to sugar content. Plant Cell Rep. 2009;28:481-91.

35. Que Y, Xu L, Wu Q, Liu Y, Ling H, Liu Y, Zhang Y, Guo J, Su Y, Chen J, et al, Genome sequencing of Sporisorium scitamineum provides insights into the pathogenic mechanisms of sugarcane smut. BMC Genomics. 2014;15:996.

36. Taniguti LM, Schaker PDC, Benevenuto J, Peters LP, Carvalho G, Palhares A, Quecine MC, Nunes FRS, Kmit MCP, Wai A, et al. Complete genome sequence of Sporisorium scitamineum and biotrophic interaction transcriptome with sugarcane. PLoS One. 2015;10:e0129318.

37. Dutheil JY, Mannhaupt G, Schweizer G, Sieber CMK, Munsterkoetter M, Gueldener U, Schirawski J, Kahmann R. A tale of genome compartmentalization: the evolution of virulence clusters in smut fungi. Genome Biol Evol. 2016;8:681-704.

38. Yan M, Dai W, Cai E, Deng YZ, Chang C, Jiang Z, Zhang L-H. Transcriptome analysis of Sporisorium scitamineum reveals critical environmental signals for fungal sexual mating and filamentous growth. BMC Genomics. 2016;17:354.

39. Heinze B, Thokoane L, Williams N, Barnes J, Rutherford R. The smutsugarcane interaction as a model system for the integration of marker discovery and gene isolation. Proc S Afr Sug Technol Ass. 2001;75:88-93.

40. Butterfield M, Rutherford R, Carson D, Huckett B, Bornman C. Application of gene discovery to varietal improvement in sugarcane. S Afr J Bot. 2004;70: $167-72$.

41. Que Y-X, Lin J-W, Song X-X, Xu L-P, Chen R-K. Differential gene expression in sugarcane in response to challenge by fungal pathogen Ustilago scitaminea revealed by CDNA-AFLP. Biomed Res Int. 2011;2011:160934.

42. Casu RE, Selivanova A, Perroux JM. High-throughput assessment of transgene copy number in sugarcane using real-time quantitative PCR. Plant Cell Rep. 2012;31(1):167-77.

43. Su Y, Xu L, Wang S, Wang Z, Yang Y, Chen Y, Que Y. Identification, phylogeny, and transcript of chitinase family genes in sugarcane. Sci Rep. 2015:5:10708.

44. Barnabas EL, Ashwin N, Kaverinathan $K$, Trentin AR, Pivato M, Sundar AR, Malathi P, Viswanathan R, Rosana OB, Neethukrishna K. Proteomic analysis of a compatible interaction between sugarcane and Sporisorium scitamineum. Proteomics. 2016:16:1111-22.

45. Pfaffl MW. A new mathematical model for relative quantification in real-time RT-PCR. Nucleic Acids Res. 2001:29:2002-7.

46. Bustin SA, Benes V, Garson JA, Hellemans J, Huggett J, Kubista M, Mueller R, Nolan T, Pfaffl MW, Shipley GL. The MIQE guidelines: minimum information for publication of quantitative real-time PCR experiments. Clin Chem. 2009;55:611-22.

47. Kwan Y-M, Meon S, Ho C-L, Wong M-Y. Selection of reference genes for quantitative real-time PCR normalization in Ganoderma-infected oil palm (Elaeis guineensis) seedlings. Australas Plant Path. 2016:45:261-8.

48. Vieira A, Cabral A, Fino J, Azinheira HG, Loureiro A, Talhinhas P, Pires AS, Varzea V, Moncada P, Oliveira H, et al. Comparative validation of conventional and rna-seq data-derived reference genes for qpcr expression studies of colletotrichum kahawae. PLoS One. 2016;11:e0150651.

49. Kozera B, Rapacz M. Reference genes in real-time PCR. J Appl Genet. 2013; 54:391.

50. Wan H, Zhao Z, Qian C, Sui Y, Malik AA, Chen J. Selection of appropriate reference genes for gene expression studies by quantitative real-time polymerase chain reaction in cucumber. Anal Biochem. 2010;399:257-61.

51. Liu D, Shi L, Han C, Yu J, Li D, Zhang Y. Validation of reference genes for gene expression studies in virus-infected Nicotiana benthamiana using quantitative real-time PCR. PLoS One. 2012;7:e46451. 
52. Chi C, Shen Y, Yin L, Ke X, Han D, Zuo Y. Selection and validation of reference genes for gene expression analysis in Vigna angularis using quantitative real-time RT-PCR. PLoS One. 2016;11:e0168479.

53. Singh V, Kaul SC, Wadhwa R, Pati PK. Evaluation and selection of candidate reference genes for normalization of quantitative RT-PCR in Withania somnifera (L.) Dunal. PLoS One. 2015;10:e0118860.

54. Xiao D, Zhang N-W, Zhao J-J, Bonnema G, Hou X-L. Validation of reference genes for real-time quantitative PCR normalisation in non-heading Chinese cabbage. Funct Plant Biol. 2012;39:342-50.

55. Camoes F, Islinger M, Guimaraes SC, Kilaru S, Schuster M, Godinho LF, Steinberg G, Schrader M. New insights into the peroxisomal protein inventory: acyl-CoA oxidases and -dehydrogenases are an ancient feature of peroxisomes. BBA-Mol Cell Res. 2015;1853:111-25.

56. Palusa SG, Reddy ASN. Extensive coupling of alternative splicing of premRNAs of serine/arginine (SR) genes with nonsense-mediated decay. New Phytol. 2010;185:83-9.

57. Reddy ASN, Ali GS. Plant serine/arginine-rich proteins: roles in precursor messenger RNA splicing, plant development, and stress responses. Wires RNA. 2011;2:875-89.

58. Hu Y, Chen H, Luo C, Dong L, Zhang S, He X, Huang G. Selection of reference genes for real-time quantitative $P C R$ studies of kumquat in various tissues and under abiotic stress. Sci Hortic-Amsterdam. 2014;174:207-16.

59. Ribeiro PR, Dekkers BJW, Fernandez LG, de Castro RD, Ligterink W, Hilhorst HWM. Identification of reference genes for gene expression studies during seed germination and seedling establishment in Ricinus communis L. Seed Sci Res. 2014;24:341-52.

60. Ye X, Zhang F, Tao Y, Song S, Fang J. Reference gene selection for quantitative real-time PCR normalization in different cherry genotypes, developmental stages and organs. Sci Hortic-Amsterdam. 2015;181:182-8.

61. Buey RM, Ledesma-Amaro R, Balsera M, Maria de Pereda J, Luis Revuelta J. Increased riboflavin production by manipulation of inosine 5'monophosphate dehydrogenase in Ashbya gossypii. Appl Microbiol Biotechnol. 2015:99:9577-89.

62. Park J-H, Ahn SH. IMP dehydrogenase is recruited to the transcription complex through serine 2 phosphorylation of RNA polymerase II. Biochem Biophys Res Commun. 2010;392:588-92.

63. Hann BC, Stirling CJ, Walter P. SEC65 gene product is a subunit of the yeast signal recognition particle required for its integrity. Nature. 1992;356:532-3.

\section{Ready to submit your research? Choose BMC and benefit from:}

- fast, convenient online submission

- thorough peer review by experienced researchers in your field

- rapid publication on acceptance

- support for research data, including large and complex data types

- gold Open Access which fosters wider collaboration and increased citations - maximum visibility for your research: over $100 \mathrm{M}$ website views per year

At BMC, research is always in progress.

Learn more biomedcentral.com/submissions 